\title{
Advanced Image Coregistration within the Leksell Workstation for the Planning of Glioma Surgery: Initial Experience
}

\author{
Manabu Tamura ${ }^{1,2}$ Motohiro Hayashi, ${ }^{1,2}$ Yoshiyuki Konishi ${ }^{1}$ Noriko Tamura $^{2}$ Jean Regis ${ }^{3}$ \\ Jean François Mangin ${ }^{4}$ Takaomi Taira ${ }^{2}$ Yoshikazu Okada ${ }^{2}$ Yoshihiro Muragaki ${ }^{1,2}$ Hiroshi Iseki ${ }^{1,2}$ \\ ${ }^{1}$ Faculty of Advanced Techno-Surgery, Institute of Advanced \\ Biomedical Engineering and Science, Tokyo Women's Medical \\ University, Tokyo, Japan \\ 2 Department of Neurosurgery, Neurological Institute, Tokyo \\ Women's Medical University, Tokyo, Japan \\ ${ }^{3}$ Department of Stereotactic and Functional Neurosurgery, Timone \\ University, Marseille, France \\ ${ }^{4}$ The Computer-Assisted Neuroimaging Laboratory, NeuroSpin, \\ Biomedical Imaging Institute, CEA, Gif sur Yvette, France \\ Address for correspondence Manabu Tamura, MD, DMSc, Faculty of \\ Advanced Techno-Surgery, Institute of Advanced Biomedical \\ Engineering and Science, Tokyo Women's Medical University, 8-1 \\ Kawada-cho, Shinjuku-ku, Tokyo 162-8666, Japan \\ (e-mail: Tamura.Manabu@twmu.ac.jp). \\ J Neurol Surg Rep 2013;74:118-122.
}

\begin{abstract}
Keywords

- Leksell GammaPlan

- Leksell SurgiPlan

- image coregistration

- diffusion tensor imaging

- gamma knife surgery

- brain tumor surgery

Background Leksell GammaPlan (LGP) and SurgiPlan (ELEKTA Instruments AB, Stockholm, Sweden) may be used effectively for the detailed evaluation of regional neuroanatomy before open neurosurgical procedures. We report our initial experience in the cases of cerebral gliomas.

Methods LGP v.8.3 was used before the surgical resection of cerebral gliomas for (1) the delineation of subdural grid electrodes and a detailed evaluation of their position relatively to cortical structures, and (2) for the fusion of structural magnetic resonance imaging and diffusion tensor imaging (DTI) for a detailed visualization of the corticospinal tract (CST) and optic radiation.

Results Delineation of the subdural grid within LGP in a patient with seizures caused by left parietal glioma permitted a detailed assessment of the location of electrodes relative to the cortical gyri and sulci and significantly facilitated interpretation of brain mapping before tumor resection. In another patient with parieto-occipital glioma, simultaneous three-dimensional visualization of the tumor, CST, and optic radiation with the use of LGP permitted us to perform tumor resection without postoperative neurologic complications. Finally, incorporation of DTI into SurgiPlan resulted in precise planning of stereotactic biopsy for bilateral thalamic glioma.

Conclusion The possibility for detailed evaluation of regional neuroanatomy based on various images within LGP and SurgiPlan may facilitate effective and safe surgical management of intracranial gliomas.
\end{abstract}

\section{Introduction}

Leksell GammaPlan (LGP; ELEKTA Instruments AB, Stockholm, Sweden) is dedicated computer-aided software that was developed for treatment planning and radiation dosimetry during gamma knife surgery (GKS). Later on, its analog SurgiPlan (ELEKTA Instruments AB) was created to facilitate stereotactic brain surgery, mainly in cases of functional brain received

February 6, 2013

accepted

August 12, 2013

published online

October 22, 2013
DOI http://dx.doi.org/

10.1055/s-0033-1358380.

ISSN 2193-6358. (c) 2013 Georg Thieme Verlag KG Stuttgart · New York
License terms

(๑) $\Theta \circledast$ 
disorders. The current versions of these systems provide extensive options for highly accurate and precise coregistration and fusion of various images (computed tomography [CT], magnetic resonance imaging [MRI], digital subtraction angiography, positron emission tomography, magnetoencephalogram, etc.) within the same workspace, their magnification and reconstruction in axial, coronal, and sagittal planes. Moreover, various anatomical structures delineated on the different images may be combined on the composite view and evaluated three-dimensionally from the different angles and directions. These capabilities can be used not only for radiosurgery or stereotactic interventions, but for planning of open neurosurgical procedures as well. Previously we successfully applied a detailed evaluation of regional neuroanatomy with the use of LGP before surgery for complex intracranial aneurysms ${ }^{1}$ and skull base tumors. ${ }^{2-4}$ Here we report our initial experience with the use of Leksell workstations for planning the surgical resection of cerebral gliomas.

\section{Methods and Materials}

LGP v.8.3 was used before the surgical resection of cerebral gliomas for (1) delineation of the subdural grid electrodes and detailed evaluation of their position relatively to cortical structures, and (2) fusion of the structural MRI and diffusion tensor imaging (DTI) for detailed visualization of the corticospinal tract (CST) and optic radiation. Several functions available within the software were used. Fusion of images obtained with various neuroimaging modalities and at different time was done with "Coregistration." Besides the main automatic algorithm, it also provides a manual mode that may be useful if correction and/or modification of the fusion technique are required. Delineation of the various intracranial anatomical structures was performed with "Regions and Volumes." "Pre-Planning" was used for fast three-dimensional (3D) delineation of the tumor with simulation of its coverage with prescription isodose. Assessment of the possible distortion artifacts on MR images was performed after their fusion with CT.

\section{Results}

\section{Evaluation of Positioning of Grid Electrodes with Regard to Cortical Anatomy}

We evaluated the positioning of grid electrodes with regard to cortical anatomy within LGP in a patient with a left parietal glioma. Manifestation of the disease with seizures and location of the lesion within the eloquent cortex led to a decision to implant chronic subdural electrodes prior to tumor resection for detailed cortical mapping.

Before the implantation of the subdural grid electrodes, volumetric MRI (slice thickness, $2.0 \mathrm{~mm}$; TE, 6.2 milliseconds [ms]; TR, 10.3 ms; flip angle, 8 degrees) was performed on a 3T Philips ACHIEVA scanner (Philips MRI Equipment, Eindhoven, The Netherlands). Matching of the cortical sulci and gyri was done with Brain VISA software based on T1-weighted MR images. ${ }^{5-7}$ After implantation of the grid, a thin-sliced bone window CT was performed. Both preimplantation MRI and postimplantation CT were incorporated into LGP. The former was used for evaluation of the cortical neuroanatomy, the latter for delineation of the subdural electrodes.

Coregistration of preimplantation MRI and postimplantation CT images permitted evaluation of the electrode position relative to the cortical gyri and sulci; use of surface anatomy scanning permitted 3D visualization of the grid position. Fusion of bone window CT and MRI revealed the presence of mild brain shift and permitted us to make a corresponding adjustment during brain mapping (-Fig. 1).

\section{Fusion of Structural Magnetic Resonance Imaging and Diffusion Tensor Imaging}

Simultaneous 3D visualization of the tumor, CST, and optic radiation with the use of LGP was applied for the planning of a parieto-occipital glioma resection (-Fig. 2) and the planning of a stereotactic biopsy for a bilateral thalamic glioma (-Fig. 3).

Volumetric MRI, as well as high signal-to-noise ratio and low-distortion DTI with 6 and 32 directions of diffusion sensitizing gradient and nearly isotropic voxel size were acquired on at 3-T MR Philips ACHIEVA scanner. Raw data

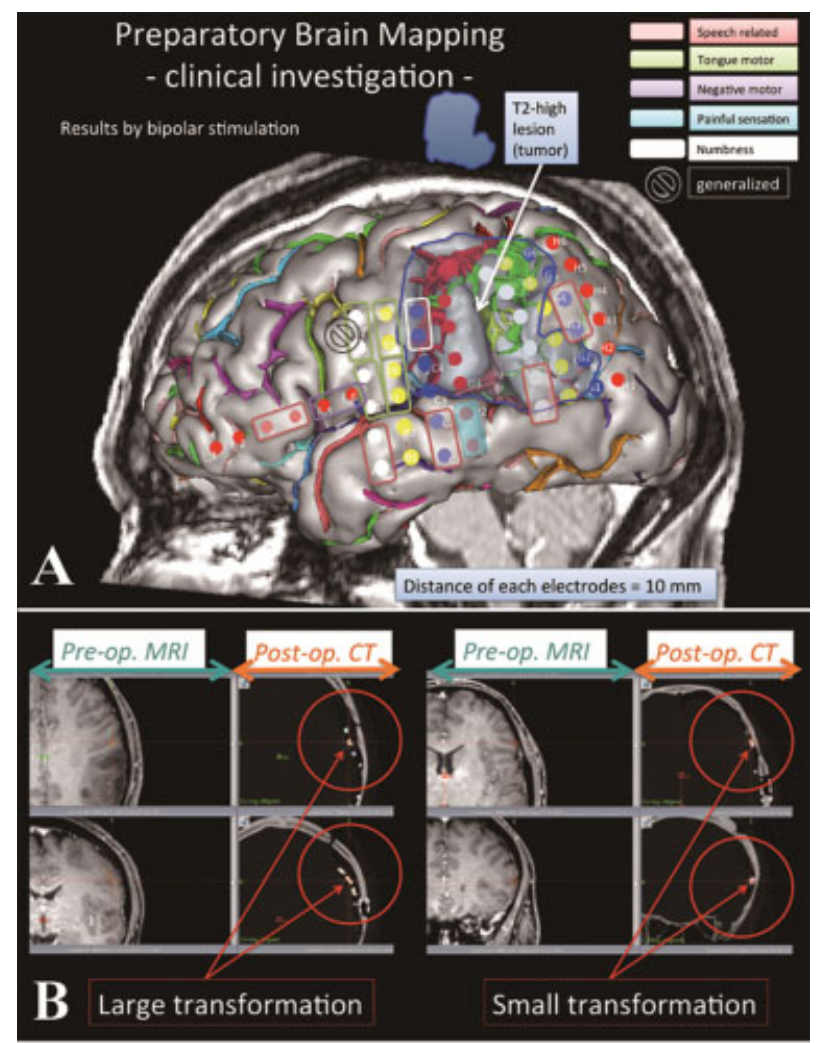

Fig. 1 Evaluation of grid electrode positioning with regard to cortical anatomy within Leksell GammaPlan in a patient with left parietal glioma. (A) Coregistration of preimplantation magnetic (MRI) and postimplantation computed tomography $(\mathrm{CT})$ images permitted evaluation of the electrode location relative to the cortical gyri and sulci. Three-dimensional visualization of the grid position was attained with surface anatomy scanning. Matching of the cortical sulci and gyri was done with Brain VISA software. (B) Fusion of bone window CT and $M R I$ revealed the presence of brain shift. 

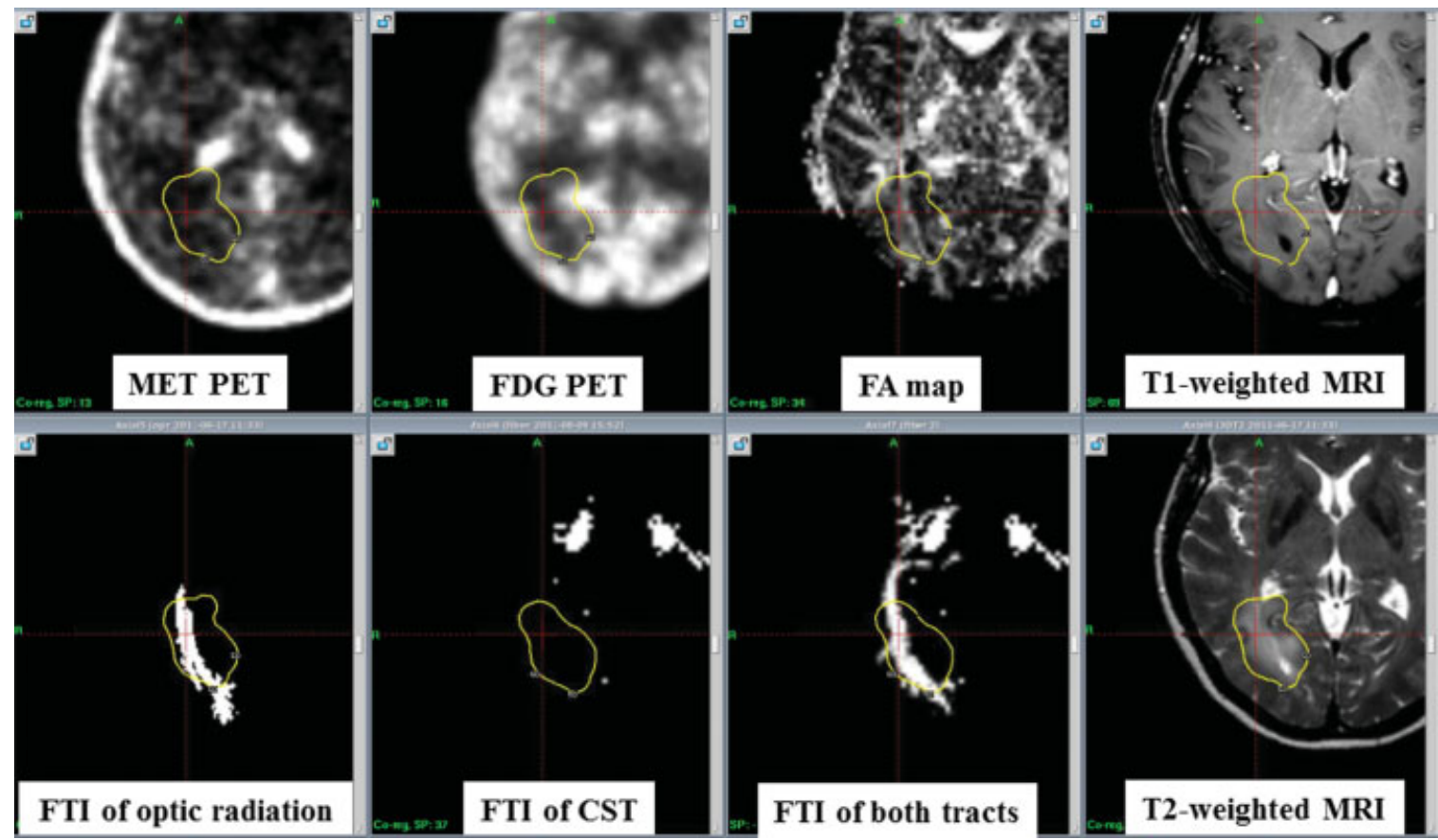

Fig. 2 Coregistration of the various images within Leksell GammaPlan for planning of the surgical resection of the left parieto-occipital glioma, Delineation of the tumor was done by simulation of its coverage with prescription isodose based on the area of hyperintensity on T2-weighted magnetic resonance imaging. CST, corticospinal tract; FA, fractional anisotropy; FDG PET, 18F-fluorodeoxyglucose positron emission tomography; FTI, fiber tracking image; MET PET, 11C-methionine positron emission tomography; MRI, magnetic resonance imaging.

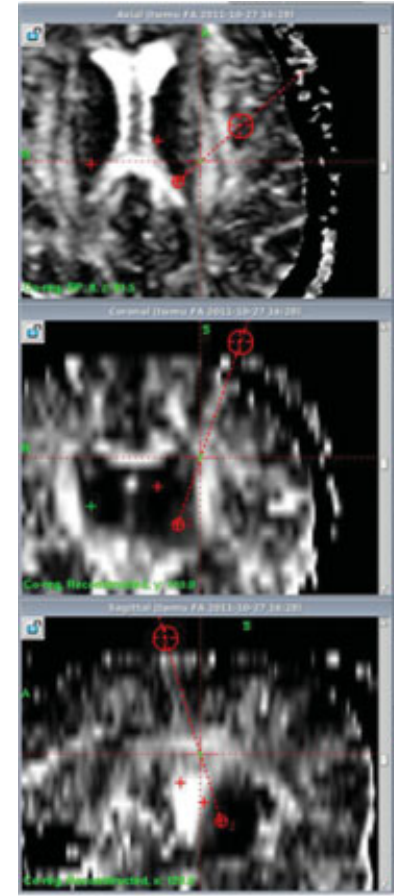

FA map

(6 directions of DSG)
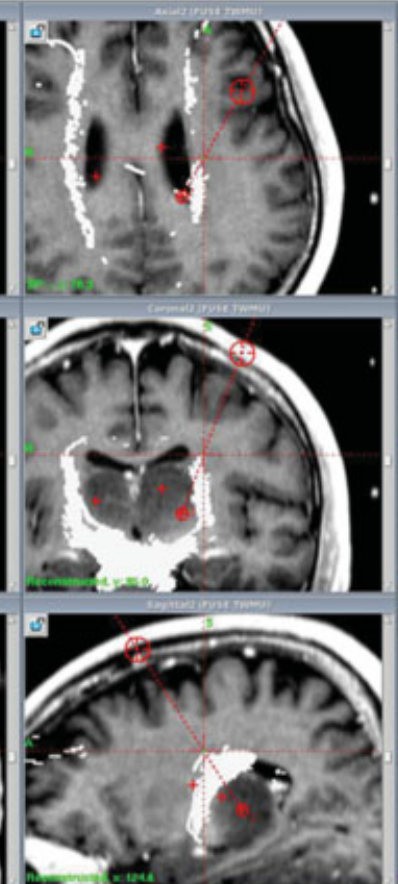

Fused FTI and MRI

(6 directions of DSG)
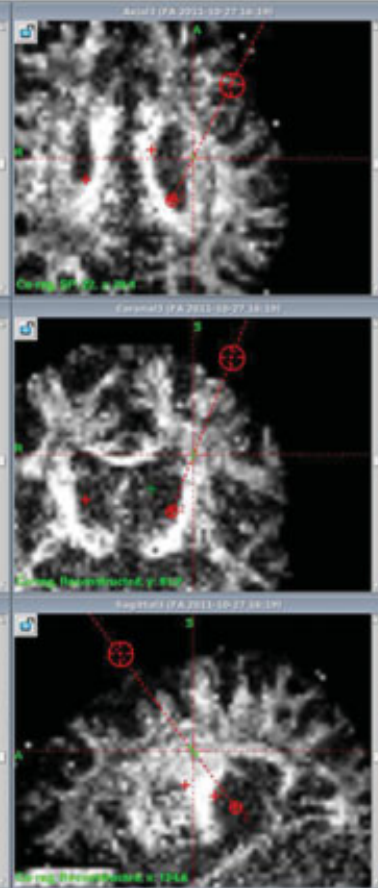

FA map

(32 directions of DSG)

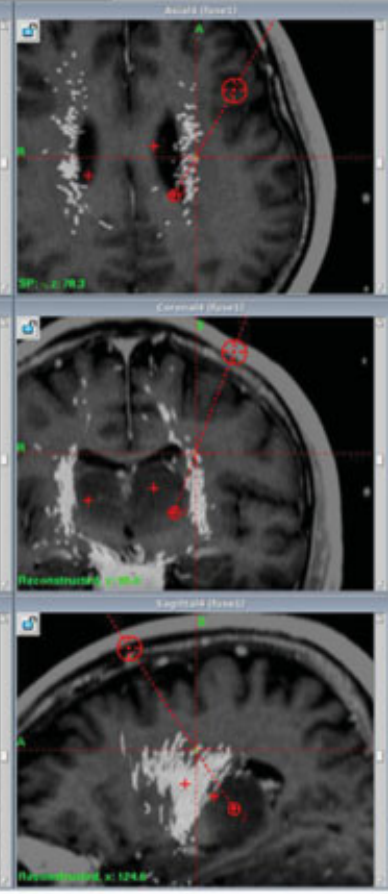

Fused FTI and MRI (32 directions of DSG)

Fig. 3 Importation of the fractional anisotropy map and fiber tracking images obtained with 6 and 32 directions of diffusion sensitizing gradient (DSG) into Leksell SurgiPlan and their three-dimensional coregistration with T1-weighted magnetic resonance imaging for stereotactic biopsy planning. Note the better resolution provided by diffusion tensor imaging obtained with 32 directions of DSG. FA, fractional anisotropy; FTI, fiber tracking image; MRI, magnetic resonance imaging. 
of DTI were analyzed with the dedicated software (Stealth DTV, Medtronic Co., USA). A fractional anisotropy (FA) map was created with tracking of the CST (region of interest [ROI] in the lower brainstem) and optic radiation (ROI in the lateral geniculate nucleus). Both the FA map and fiber tracking images were exported in the 3D volumetric Digital Imaging and Communications in Medicine (DICOM) format into LGP independently from each other, and they underwent coregistration with T1- and T2-weighted MR images using an automatic setting (for the FA map) and a manual one (for fiber tracking images). Subsequently, delineation of both CST and optic radiation was done. The lesion itself was visualized by simulation of the radiosurgical procedure based on location of the hyperintense area on T2-weighted MR images.

In both cases, fusion of the structural MRI and DTI facilitated surgical manipulations, which were not accompanied by any postoperative deterioration of motor and visual functions.

\section{Discussion}

Various functions available within Leksell workstations (LGP and SurgiPlan) permit safe and effective treatment planning for GKS and stereotactic neurosurgery. For example, precise delineation of the functionally important anatomical structures leads to avoidance of their excessive irradiation, fusion of bone window CT and MRI provides an opportunity to estimate and correct distortion artifacts, and evaluation of the target area on coregistered images significantly facilitates the differential diagnosis among various neoplasms with the same location (e.g., meningioma versus schwannoma) and the precise identification of the tumor origin from the specific dural area or cranial nerve. ${ }^{3,8}$ Moreover, 3D visualization of the various structures adjacent to the lesion and the possibility of their evaluation from different angles and directions permit the surgeon to simulate a view of the surgical field and may be very useful for the planning of open neurosurgical procedures, which may result in greater effectiveness, lower morbidity, and a better outcome. ${ }^{4}$ Therefore, we have recently begun to use LGP for the evaluation of complex regional neuroanatomy prior to removal of skull base tumors and the clipping of cerebral aneurysms. ${ }^{1-4}$ Our initial experience reported here shows that a similar technique can also be applied effectively in cases of parenchymal brain tumors such as gliomas.

The originally created mapping of subdural electrodes permits a precise evaluation of their location and adjusts the results of cortical mapping for the possible presence of brain shift. Because electrodes could not be seen well on MR images, it was decided to perform their coregistration with a thin-sliced bone window CT obtained after grid implantation. ${ }^{9}$ Although it was effectively accomplished in the presented case, the possible limitation of the technique should be mentioned. Theoretically, because the brain surface is especially susceptible to shift after craniotomy, ${ }^{10}$ the delineated electrodes on postoperative CT scan may not correspond well to the cortical anatomy visualized with the preimplantation MRI. Therefore, our proposed method requires further vali- dation and at present should be considered a useful adjunct but not a substitution for intraoperative brain mapping with direct electrical stimulation of the cortex. ${ }^{11,12}$

Contemporary MRI-based neuroimaging analysis plays an important part in the neurosurgical field. Mainly, T1- and T2weighted MRI offers detailed information on the location and pathology of the brain. ${ }^{13}$ Nevertheless, at present, MRI provides variable functional and metabolic data. Particularly, use of DTI (FA and fiber tracking images) is very effective for the evaluation of white matter tracts. ${ }^{14}$ It has been successfully applied for GKS of arteriovenous malformations and appears to increase the safety of radiosurgical procedures. ${ }^{15-17}$ In our experience, incorporation of FA maps and fiber tracking images in LGP may permit precise planning of resection for gliomas located in the eloquent brain areas. Nevertheless, additional studies are necessary for the evaluation of possible distortions and correspondence of DTI to the actual anatomical tract position. In particular, the optimal location of the ROI for seed/target should be defined and appropriateness of the deterministic tracking method should be confirmed. This preliminary importation of DTI in LGP and SPS has the potential to be used extensively for safe GKS procedures and stereotactic neurosurgery, respectively.

\section{Conclusions}

Leksell computer-aided workstations LGP and SurgiPlan may be helpful for planning brain tumor resection. The possibility for a detailed evaluation of regional neuroanatomy based on various images, including DTI, may help avoid postoperative neurologic deterioration.

\section{Acknowledgments}

This study was supported by Grant-in-Aid for Scientific Research (B) 22300093 and was done under the auspices of the Global COE Program, namely the Multidisciplinary Education and Research Centre for the Establishment of Regenerative Medicine (MERCREM). Additional support was obtained from JST and CREST. The authors do not have any personal or institutional financial interests in drugs, materials, or devices described in this article. We thank Drs. Gautam A. Deshpande and Mikhail Chernov for their help with the preparation of the manuscript.

\section{Note}

The results of this study were presented during the 16th International Meeting of the Leksell Gamma Knife Society (March 25-29, 2012; Sydney, Australia).

\section{References}

1 Kawashima A, Okada Y, Hayashi M, Kawamata T, Hori T. Application of contrast-enhanced constructive interference in steady state magnetic resonance imaging to Leksell GammaPlan for localizing c2-c3 aneurysms: technical note. Neurosurgery 2009;65(6): E1188-E1190; discussion E1190 
2 Obara T, Hayashi M, Ino H, et al. Surgical management of skull base meningiomas: a treatment strategy using multi-modality [in Japanese]. Progress in CI 2009;31(2):73-81

3 Hayashi M, Chernov MF, Tamura N, et al. Usefulness of the advanced neuroimaging protocol based on plain and gadolinium-enhanced constructive interference in steady state images for gamma knife radiosurgery and planning microsurgical procedures for skull base tumors. Acta Neurochir Suppl 2013;116: 167-178

4 Tamura M, Konishi Y, Tamura N, et al. Usefulness of Leksell GammaPlan for preoperative planning of brain tumor resection: delineation of the cranial nerves and fusion of the neuroimaging data, including DTI. Acta Neurochir Suppl 2013;116:179-185

5 Mangin JF, Rivière D, Cachia A, et al. Object-based morphometry of the cerebral cortex. IEEE Trans Med Imaging 2004;23(8):968-982

6 Mangin JF, Rivière D, Cachia A, et al. A framework to study the cortical folding patterns. Neuroimage 2004;23(Suppl 1):S129-S138

7 Regis J, Tamura M, Park MC, et al. Subclinical abnormal gyration pattern, a potential anatomical marker of epileptogenic zone in patients with MRI-negative frontal lobe epilepsy. Neurosurgery 2011;69(1):80-94; discussion 93-94

8 Hayashi M, Chernov M, Tamura N, et al. Gamma Knife robotic microradiosurgery of pituitary adenomas invading the cavernous sinus: treatment concept and results in 89 cases. J Neurooncol 2010;98(2):185-194

9 Tamura M, Nishibayashi H, Kakishita K, et al. Preoperative MRIbased delineation of the central sulcus and its usefulness for the intraoperative navigation of the epidural electrodes implantation for the motor cortex stimulation [in Japanese]. Funct Neurosurg 2010;49(2):201-207
10 Ozawa N, Muragaki Y, Nakamura R, Hori T, Iseki H. Shift of the pyramidal tract during resection of the intraaxial brain tumors estimated by intraoperative diffusion-weighted imaging. Neurol Med Chir (Tokyo) 2009;49(2):51-56

11 Ozawa N, Muragaki Y, Nakamura R, Iseki H. Identification of the pyramidal tract by neuronavigation based on intraoperative diffusion-weighted imaging combined with subcortical stimulation. Stereotact Funct Neurosurg 2009;87(1):18-24

12 Giussani C, Roux FE, Ojemann J, Sganzerla EP, Pirillo D, Papagno C. Is preoperative functional magnetic resonance imaging reliable for language areas mapping in brain tumor surgery? Review of language functional magnetic resonance imaging and direct cortical stimulation correlation studies. Neurosurgery 2010;66(1): 113-120

13 Muragaki Y, Iseki H, Maruyama T, et al. Information-guided surgical management of gliomas using low-field-strength intraoperative MRI. Acta Neurochir Suppl 2011;109:67-72

14 Nimsky C, Ganslandt O, Hastreiter P, et al. Preoperative and intraoperative diffusion tensor imaging-based fiber tracking in glioma surgery. Neurosurgery 2005;56(1):130-137; discussion 138

15 Maruyama K, Koga T, Kamada K, et al. Arcuate fasciculus tractography integrated into Gamma Knife surgery. J Neurosurg 2009; 111(3):520-526

16 Koga T, Maruyama K, Kamada K, et al. Outcomes of diffusion tensor tractography-integrated stereotactic radiosurgery. Int J Radiat Oncol Biol Phys 2012;82(2):799-802

17 Koga T, Shin M, Maruyama K, et al. Integration of corticospinal tractography reduces motor complications after radiosurgery. Int J Radiat Oncol Biol Phys 2012;83(1):129-133 